\title{
The Effects of Different Exercise Modalities on s-Klotho after Surgery: A Call for Action
}

\section{Moran S Saghiv*}

Exercise Physiology Department, University of Mary, North Dakota, USA

Although phrased in many different ways, it has been known for many years that different modalities of exercise may have a positive influence on the extent and pace of recovery from different types of surgery. Now days this idea is termed "Exercise is Medicine", and has great relevancy to post-surgery recovery through exercising.

Klotho gene expression in positively influenced by different exercise modalities acting as an epigenetic influencer [1], resulting in increased work capacity, performance, and lifespan, and decreased aging in healthy and diseased populations [2-4]. As is the case with exercise, positive adaptations decrease and diminish with time if exercise training is not maintained.

Klotho is a transmembrane protein that provides some control over the sensitivity of the organism to insulin and appears to be involved in aging [5]. Age-related declines are manifest by a decreased ability for aged skeletal muscle to respond to physiological stimuli such as muscle loading or acute injury, and disease related effects [6,7].

Regular aerobic exercise participation promotes health and disease prevention [8].

Endurance exercise like biking, walking, swimming and running, appear to benefit longer life expectancy than anaerobic exercise like power lifting [9]. It has been suggested that circulating Klotho levels are upregulated in response to an acute exercise bout, but that the response may be dependent on fitness level [10-13].

Compared to sedentary young and old subjects, in the elite well aerobic trained young runners and master athletes s-Klotho levels are markedly elevated while, IGF-I levels were decreased [14]. IGF-I is generally thought to be associated with anabolism and wellbeing [15], yet, signaling through IGF-I and Insulin receptors is negatively related to adults [16]. A meta-analysis study indicated that increased circulating concentrations of IGF-I is associated with increased risks for colorectal, prostate, and premenopausal breast cancers [17].

Several clinical studies have suggested that Klotho gene exerts strong cardio-protective effects. S-Klotho has been proposed as a key regulator of the development of cardiovascular disease. An association between low levels of s-Klotho and the occurrence and severity of cardiovascular disease has been reported, as well as a reduction of cardiovascular risk when levels were high [17]. This protein is related to the attenuation of vascular calcification as well as prevention of cardiac hypertrophy.

In recent years, studies have been conducted in order to study the effects of different types of surgery on s-Klotho in a variety of research designs.

Kakareko et al. [18] concluded that "Nephrectomy among patients with preserved renal function before surgery does not increase c-FGF-23 but reduces sKL. Moreover, nephrectomy results in derangements in bone turnover markers in short-term follow-up. These changes may participate in pathogenesis of bone disease after nephrectomy".
Liao et al. [19] reported as part of their study's conclusions that "There was no change in circulating FGF23 and Klotho concentrations after PTX in hemodialysis patients given postoperative calcium supplements and/or vitamin D analogue. Serum FGF23 concentrations pre-PTX and at days 5 and 90 after PTX were inversely related to serum calcium concentrations".

Kimura et al. [20] concluded that "The current data regarding the urinary soluble Klotho in recipients support the hypothesis that the kidney is a major source of urinary soluble Klotho among the numerous components of the urinary tract. In living donors, the complex nature of events associated with acute reductions in the renal mass may modulate the release of soluble Klotho from the kidneys into the urine".

Takahashi et al. [21] while aiming to "determine the impact of parathyroidectomy $[\mathrm{PTx}]$ on serum FGF23 and soluble Klotho levels in patients with severe secondary hyperparathyroidism" suggested that "the parathyroid gland is not the major site of soluble Klotho production in patients with end-stage renal disease, and the production of Klotho by other organ(s) is affected by alterations in mineral metabolism or medications taken after PTx".

Kohler, et al. [22] concluded that "Soluble Klotho could be useful in the follow-up of acromegalic patients. The question arises whether s-Klotho not only reflects the activity of GH-secreting pituitary adenomas but whether Klotho (ectodomain clipping?) could also mediate selected actions of GH" while GHR genotyping 112 patients with acromegaly after transsphenoidal surgery.

While s-Klotho is being researched more and more as of late, to the best of the author's knowledge, very few data exist that has been published in peer reviewed journals pertaining to the influence of exercise on s-Klotho, an "emerging" new biomarker of anti-aging and health, evermore so when it pertains to the influence of post-surgery exercising on this biomarker.

As both data pertaining to the role of surgery of different types on s-Klotho and data pertaining to the influence of exercising post-surgery on s-Klotho are limited, it is the wish of the author via this editorial to call for more research to be conducted in order to establish an evergrowing pool of knowledge regarding the influences of surgery and post-surgery exercising on s-Klotho.

*Corresponding author: Dr. Moran S Saghiv, Associate Professor, Exercise Physiology Department, University of Mary, North Dakota, USA, Tel: 702-908-2390; E-mail: mssaghiv@umary.edu

Received February 04, 2017; Accepted February 17, 2017; Published February 24, 2017

Citation: Saghiv MS (2017) The Effects of Different Exercise Modalities on s-Klotho after Surgery: A Call for Action. J Vasc Med Surg 5: 303. doi: 10.4172/23296925.1000303

Copyright: @ 2017 Saghiv MS. This is an open-access article distributed under the terms of the Creative Commons Attribution License, which permits unrestricted use, distribution, and reproduction in any medium, provided the original author and source are credited. 
Citation: Saghiv MS (2017) The Effects of Different Exercise Modalities on s-Klotho after Surgery: A Call for Action. J Vasc Med Surg 5: 303. doi: 10.4172/2329-6925.1000303

Page 2 of 2

\section{References}

1. Ling C, Ronn T (2014) Epigenetic Adaptation to Regular Exercise in Humans. Drug Discov Today 19: 1015-1018.

2. Denham J, Marques FZ, O’Brien BJ, Charchar FJ (2014) Exercise: Putting Action into Our Epigenome. Sports Med 44: 189-209.

3. Bian A, Neyra JA, Zhan M, Klotho HMC (2015) Stem Cells, and Aging. Clin Interv Aging 10: 1233-1243.

4. Matsubara T, Miyaki A, Akazawa N, Choi Y, Ra SG, et al. (2014) Aerobic Exercise Training Increases Plasma Klotho Levels and Reduces Arterial Stiffness in Postmenopausal Women. Am J Physiol Heart Circ Physiol 306: H348-H355.

5. Kuro-o M, Matsumura Y, Aizawa H, Kawaguchi H, Suga T, et al. (1997) Mutation of the Mouse Klotho Gene Leads to a Syndrome Resembling Ageing. Nature 390: 45-51.

6. Wang Y, Sun Z (2009) Current Understanding of Klotho. Ageing Res Rev 8: 43-51.

7. Wang Y, Sun Z (2014) Antiaging Gene Klotho Regulates Endothelin-1 Levels and Endothelin Receptor Subtype B Expression in Kidneys of Spontaneously Hypertensive Rats. Journal of Hypertension 32: 1629-1636.

8. Paffenbarger RS, Blair SN, Lee IM (2001) A History of Physical Activity, Cardiovascular Health and Longevity: The Scientific Contributions of Jeremy N Morris, DSc, DPH, FRCP. Int J Epidemiol 30: 1184-1192.

9. Schefer V, Talan MI (1996) Oxygen Consumption in Adult and AGED C57BL/6 Mice during Acute Treadmill Exercise of Different Intensity. Exp Gerontol 31: 387-392.

10. Phelps M, Pettan-Brewer C, Ladige W, Yablonka-Reuveni Z (2013) Decline in Muscle Strength and Running Endurance in Klotho Deficient C57BL/6 Mice. Biogerontology 14: 729-739.

11. Saghiv M, Goldhammer E, Sagiv M, Ben Sira D (2015) Effects of Aerobic Exercise Training on S-Klotho in Young and Elderly. J Physiology 1: 001.

12. Lee EY, Kim SS, Lee JS (2014) Soluble $\alpha$-Klotho as a Novel Biomarker in the Early Stage of Nephropathy in Patients with Type 2 Diabetes. PLoS ONE 9: e102984.
13. Bonnefoy M, Patricot MC, Lacour JR, Rahmani A, Berthouze S, et al. (2002) Relation Between Physical Activity, Muscle Function and IGF-I, Testosterone and DHEAS Concentrations in the Elderly. Rev Med Interne 23: 819-827.

14. Berryman DE, Christiansen JS, Johannsson G, Thorner MO, Kopchick JJ (2008) Role of the GH IGF-I Axis in Lifespan and Health Span: Lessons from Animal Models. Growth Horm IGF Res 18: 455-471.

15. Ahima RS, Prabakaran D, Mantzoros C, Qu D, Lowell B, et al. (1996) Role of Leptin in the Neuroendocrine Response to Fasting. Nature 1996 382: 250-252.

16. Chateau MT, Araiz C, Descamps S, Galas S (2010) Klotho Interferes with a Novel FGF-Signaling Pathway and Insulin/IGF-Like Signaling to Improve Longevity and Stress Resistance in Caenorhabditis Elegans. Aging 2: 567-581.

17. Martín-Núñez E, Donate-Correa J, Muros-de-Fuentes M, Mora-Fernández C Navarro-González JF (2014) Implications of Klotho in Vascular Health and Disease. World J Cardiol 6: 1262-1269.

18. Kakareko K, Rydzewska-Rosolowska A, Brzosko S, Gozdzikiewicz-Lapinska J, Koc-Zorawska E, et al. (2017) The effect of nephrectomy on Klotho, FGF-23 and bone metabolism. Int Urol Nephrol 49: 681-688.

19. Liao SC, Moi SH, Chou FF, Yang CH, Chen JB (2016) Changes in Serum Concentrations of Fibroblast Growth Factor 23 and Soluble Klotho in Hemodialysis Patients after Total Parathyroidectomy. Biomed Res Int 2016: 6453803.

20. Kimura T, Akimoto T, Watanabe Y, Kurosawa A, Nanmoku K, et al. (2015) Impact of Renal Transplantation and Nephrectomy on Urinary Soluble Klotho Protein. Transplant Proc 47: 1697-1699.

21. Takahashi H, Komaba H, Takahashi Y, Sawada K, Tatsumi R, et al. (2014) Impact of parathyroidectomy on serum FGF23 and soluble Klotho in hemodialysis patients with severe secondary hyperparathyroidism. J Clin Endocrinol Metab 99: E652-8.

22. Kohler S, Tschopp O, Sze L, Neidert M, Bernays RL, et al. (2013) Monitoring for potential residual disease activity by serum insulin-like growth factor 1 and soluble Klotho in patients with acromegaly after pituitary surgery: is there an impact of the genomic deletion of exon 3 in the growth hormone receptor [d3 GHR] gene on "safe" GH cut-off values? Gen Comp Endocrinol 188: 282-287. 